\title{
Master of Modern Physics
}




\section{PRINCETON SERIES IN PHYSICS}

Edited by Sam B. Treiman (published since 1976)

Studies in Mathematical Physics: Essays in Honor of Valentine Bargmann edited by Elliot H. Lieb, B. Simon, and A. S. Wightman

Convexity in the Theory of Lattice Gases by Robert B. Israel

Works on the Foundations of Statistical Physics by N. S. Krylov

Surprises in Theoretical Physics by Rudolf Peierls

The Large-Scale Structure of the Universe by P.J.E. Peebles

Statistical Physics and the Atomic Theory of Matter, From Boyle and Newton to Landau and Onsager by Stephen G. Brush

Quantum Theory and Measurement edited by John Archibald Wheeler and Wojciech Hubert Zurek

Current Algebra and Anomalies by Sam B. Treiman, Roman Jackiw, Bruno Zumino, and Edward Witten

Quantum Fluctuations by E. Nelson

Spin Glasses and Other Frustrated Systems by Debashish Chowdhury

(Spin Glasses and Other Frustrated Systems is published in co-operation with World Scientific Publishing Co. Pte. Ltd., Singapore.)

Weak Interactions in Nuclei by Barry R. Holstein

Large-Scale Motions in the Universe: A Vatican Study Week edited by Vera C. Rubin and George V. Coyne, S.J.

Instabilities and Fronts in Extended Systems by Pierre Collet and Jean-Pierre Eckmann

More Surprises in Theoretical Physics by Rudolf Peierls

From Perturbative to Constructive Renormalization by Vincent Rivasseau

Supersymmetry and Supergravity (2nd ed.) by Julius Wess and Jonathan Bagger

Maxwell's Demon: Entropy, Information, Computing edited by Harvey S. Leff and Andrew F. Rex

Introduction to Algebraic and Constructive Quantum Field Theory by John C. Baez, Irving E. Segal, and Zhengfang Zhou

Principles of Physical Cosmology by P.J.E. Peebles

Scattering in Quantum Field Theories: The Axiomatic and Constructive Approaches by Daniel lagolnitzer

QED and the Men Who Made It: Dyson, Feynman, Schwinger, and Tomonaga by Silvan S. Schweber

The Interpretation of Quantum Mechanics by Roland Omnès

Gravitation and Inertia by Ignazio Ciufolini and John Archibald Wheeler

The Dawning of Gauge Theory by Lochlainn O'Raifeartaigh

The Theory of Superconductivity in the High- $T_{C}$ Cuprates by $P . W$. Anderson Master of Modern Physics: The Scientific Contributions of H. A. Kramers by D. ter Haar 


\title{
Master of Modern Physics
}

\section{The Scientific Contributions}

\author{
of H. A. Kramers \\ D. TER HAAR
}

Princeton Series in Physics 
Copyright (C) 1998 by Princeton University Press

Published by Princeton University Press, 41 William Street,

Princeton, New Jersey 08540

In the United Kingdom: Princeton University Press, Chichester, West

Sussex

\section{All Rights Reserved}

\section{Library of Congress Cataloging-in-Publication Data}

Haar, D. ter.

Master of modern physics : the scientific contributions of H.A. Kramers /

Dirk ter Haar.

p. cm. - (Princeton series in physics)

Includes bibliographical references and index.

ISBN 0-691-02141-4 (cloth : alk. paper)

1. Kramers, Hendrik Anthony, 1894-1952. 2. Quantum theory-History.

3. Physicists-Netherlands-Biography. I. Title. II. Series.

QC16.K69H33 1998

$530.12-\mathrm{dc} 21 \quad 97-22356$

The publisher would like to acknowledge the author of this volume for providing the camera-ready copy from which this book was printed

Princeton University Press books are printed on acid-free paper and meet the guidelines for permanence and durability of the Committee on Production Guidelines for Book Longevity of the Council on Library Resources

http://pup.princeton.edu

Printed in the United States of America

$\begin{array}{llllllllll}10 & 9 & 8 & 7 & 6 & 5 & 4 & 3 & 2 & 1\end{array}$ 